\title{
Valores espirométricos en pre-escolares sanos
}

\author{
Spirometric values in healthy preschool children
}

\author{
Ana Moya Olivares ${ }^{\mathrm{a}}$, Luis Villarroel del Pino ${ }^{\mathrm{b}}$, Laura Fierro Tolosa ${ }^{\mathrm{c}}$, \\ Catalina Foncea Fierro ${ }^{d}$, Solange Caussade Larraín ${ }^{a}$
}

\begin{abstract}
aDepartamento Cardiología y Enfermedades Respiratorias Pediátricas, Facultad de Medicina, Pontificia Universidad Católica de Chile bDepartamento Salud Pública, Facultad de Medicina, Pontificia Universidad Católica de Chile 'Red UC Christus, Hospital Clínico, División de Pediatría, Pontificia Universidad Católica de Chile dEscuela de Medicina, Universidad Mayor, Santiago, Chile
\end{abstract}

Recibido el 11 de junio de 2018; aceptado el 5 de noviembre de 2018

\begin{abstract}
Resumen
Introducción: La espirometría es el examen más utilizado para evaluar función pulmonar. Para niños pre-escolares actualmente se cuenta con patrones de referencia extranjeros. Objetivos: 1. Medir variables espirométricas en pre-escolares chilenos sanos, 2. Comparar estos resultados con predictivos según GLI (Global Lung Initiative), Eigen (USA) y França (Brasil) y 3. De haber diferencia significativa con éstos, elaborar ecuaciones de referencia. Sujetos y Método: Se distribuyeron encuestas a apoderados de colegios y jardines infantiles de Santiago. Se excluyeron niños con antecedente de prematurez, síntomas asmáticos, enfermedad pulmonar crónica (incluida asma) y enfermedad crónica no respiratoria. La espirometría se realizó según guías ATS/ERS 2007, con equipo MedGraphics, USA. Se registraron antecedentes familiares, ambientales, peso y talla y los valores obtenidos en capacidad vital forzada (CVF), volumen espirado forzado en $0,5,0,75$ y 1 segundo $\left(\mathrm{VEF}_{0,5}, \mathrm{VEF}_{0,75} \mathrm{y}\right.$ $\mathrm{VEF}_{1}$, respectivamente). Resultados: Se realizaron 276 espirometrías, de las cuales 202 cumplieron criterios de aceptabilidad, 112 mujeres, edad promedio 5,01 $\pm 0,57$ años, talla 108,7 $\pm 5,6 \mathrm{~cm}$. Al comparar por género, solo hubo diferencia significativa en CVF, siendo superior en hombres. Los valores promedio obtenidos en el grupo total fueron: CVF 1,22 $\pm 0,22$ litros, $\mathrm{VEF}_{1} 1,16 \pm 0,18$ litros, $\mathrm{VEF}_{0,75} 1,07 \pm 0,17$ litros. Nuestros parámetros fueron porcentualmente mayores que los predictivos según GLI, Eigen y França, excepto CVF con Eigen, motivo por el cual se construyeron ecuaciones predictivas. Conclusiones: Los valores espirométricos de pre-escolares residentes en Santiago fueron superiores a los valores de referencia extranjeros. Se proponen patrones de referencia que podrían ser utilizados en nuestro medio.
\end{abstract}

Palabras clave: Espirometría; pre-escolares; valores de referencia; función pulmonar

Correspondencia:

Solange Caussade

solangecaussade@gmail.com 


\section{Abstract}

Introduction. Spirometry is the most commonly used test to evaluate lung function. Foreign reference standards are currently available for preschool children. Objectives: 1 . To measure spirometric variables in healthy Chilean preschool children, 2. To compare these results with predictive ones according to GLI (Global Lung Initiative), Eigen (USA) and França (Brazil), and 3. If there is a significant difference with these, to develop reference equations. Subjects and Method: Questionnaires were distributed to parents in several schools and kindergartens in Santiago. Children with a history of prematurity, asthmatic symptoms, chronic lung disease (including asthma), and chronic nonrespiratory disease were excluded. Spirometry was performed according to ATS/ERS 2007 guidelines, with MedGraphics equipment, USA. Family and environmental background, weight and height were recorded, as well as values obtained in forced vital capacity (FVC), forced expiratory volume in 0.5, 0.75 and 1 second (FEV0.5, FEV0.75, and FEV1, respectively). Results: 276 spirometries were performed, 202 met acceptability criteria, 112 girls, average age $5.01 \pm 0.57$ years, height $108.7 \pm 5.6$ $\mathrm{cm}$. When comparing by gender, there was only a significant difference in FVC, which was higher in boys. The average values obtained in the total group were: FVC $1.22 \pm 0.22$ liters, FEV1 $1.16 \pm 0.18$ liters, FEV0.75 $1.07 \pm 0.17$ liters. These parameters were higher in percentage than the predictive ones according to GLI, Eigen, and França, except FVC with Eigen, therefore, predictive equations were developed. Conclusions: Spirometric values of preschoolers living in Santiago were higher than foreign reference values. We proposed these reference standards to be used in our country.

\section{Keywords:}

Spirometry; preschoolers; reference values; lung function

\section{Introducción}

La espirometría es el método más utilizado para evaluar la función pulmonar ${ }^{1-5}$. El año 2007 se publica el consenso de expertos de la Sociedad Americana de Tórax (ATS) y Sociedad Europea de Respiratorio (ERS), con las recomendaciones para su realización e interpretación en edad pre-escolar': se plantean criterios de aceptabilidad y repetibilidad propios para esta edad, fundamentados en su menor volumen pulmonar, y en la desproporción entre tamaño de la vía aérea en relación a este menor volumen pulmonar ${ }^{4-6}$. Algunos autores ya han evidenciado buenos resultados al aplicar estos nuevos criterios ${ }^{4-11}$.

En las guías referidas se discute la forma más adecuada de escoger valores de referencia, destacando que los valores realizados en sujetos mayores de 6 años no deben ser extrapolados a pre-escolares. Si se cuenta con valores obtenidos en sujetos de distintas características se deben validar estudiando al menos 300 individuos sanos. Además se hace énfasis en que los resultados deben ser expresados como z score ${ }^{1,12,13}$.

Los valores de referencia publicados a la fecha han sido obtenidos principalmente en poblaciones caucásicas, europeas o norteamericanas ${ }^{2,4,14-17}$, las cuales presentan diferencias étnicas, nutricionales y de constitución corporal con respecto a nuestra población.

Nuestra hipótesis es que los valores espirométricos de pre-escolares chilenos sanos son mayores que los valores de referencia predichos según fórmulas extranjeras. Los objetivos son: 1) Medir volúmenes y flujos espiratorios forzados; 2) Compararlos con los valores teóricos de Global Lung Initiative $(\mathrm{GLI})^{14}$, Eigen et $\mathrm{al}^{2} \mathrm{y}$ França et $\mathrm{al}^{10}$; 3) Si los valores obtenidos difieren significativamente, elaboración de ecuaciones de referencia.

\section{Sujetos y Método}

\section{Participantes}

Se contactaron directores de 13 establecimientos educacionales (colegios y jardines infantiles) ubicados en distintas comunas de Santiago. Los niños pertenecieron a 23 comunas. Según nivel socioeconómico definido por encuesta CASEN un 19,3\% de ellos vivía en comunas de ingreso alto, $49,5 \%$ ingreso medio y $31,2 \%$ ingreso bajo. Todos fueron chilenos, de padres chilenos. Se enviaron cuestionarios a los apoderados de alumnos entre 3 y 5 años 11 meses 29 días solicitando antecedentes perinatales, mórbidos generales y respiratorios, familiares y ambientales. Los criterios de exclusión fueron: prematurez (recién nacido $\leq 37$ semanas), peso al nacer $\leq 2,5 \mathrm{~kg}$, enfermedad pulmonar crónica (asma bronquial, displasia broncopulmonar, fibrosis quística, bronquiolitis obliterante, malformaciones de la vía aérea y pulmón), antecedente de hospitalización por causa respiratoria (distress neonatal, neumonía), uso de medicamentos inhalados permanentes o intermitentes, enfermedad crónica no respiratoria (neuromuscular, cardiaca, renal). Se aceptaron niños (as) con síntomas respiratorios altos leves, sin tos que les impidiera realizar el esfuerzo espiratorio para el procedimiento ${ }^{18}$. Los niños no tuvieron preparación previa ni se les entrenó para realizar un segundo intento al no lograr curvas aceptables en su primera citación. 


\section{Procedimiento}

Se midió talla con estadiómetro Seca ${ }^{\circledR}$ modelo 213 portátil, España, de pie, sin calzado y peso (pesa Medisana ${ }^{\circledR}$ Analoge Personenwaage PSD, Alemania). Se calculó índice de masa corporal (IMC), definiendo sobrepeso un IMC en rango > P85 - < P95 y obesidad IMC $\geq$ P95.

Tres operadores (AM, LF, SC) realizaron la espirometría. El equipo utilizado fue Medical Graphics Modelo CPF-S/D USB con neumotacógrafo tipo PITOT, calibrado con jeringa de 3 litros en cada visita, ajustado según temperatura, humedad y presión barométrica de la sala habilitada para el procedimiento. Se revisó linealidad de las mediciones de calibración. Los niños eran acompañados por un auxiliar de párvulos o parvularia, en grupos de 2 a 3 . Se dedicó un máximo de 15 minutos a cada niño. Se realizó la espirometría en posición de pie, inicialmente con pinza nasal y sin incentivo. En los casos en que la boquilla le ocasionaba incomodidad, se agregó un adaptador. Si el niño presentaba dificultad para realizar 3-4 maniobras iniciales se usó incentivo del software y/o retiro de la pinza nasal.

Cada niño realizó al menos 3 esfuerzos y un máximo de 12. Se observó el Volumen de extrapolación retrógrada (VER) informado por el software y se midió con regla milimetrada el tiempo espiratorio. Se determinó repetibilidad de CVF y VEF1 $\left(\mathrm{VEF}_{0,5} \mathrm{o} \mathrm{VEF}_{0,75}\right.$ si no se obtenía $\mathrm{VEF}_{1}$ ), considerando una variabilidad $<10 \%$ en los casos en que se obtenía 2 o más esfuerzos aceptables.

Los valores espirométricos registrados fueron: $\mathrm{Ca}-$ pacidad vital forzada (CVF), Volúmenes espirados forzados $\left(\mathrm{VEF}_{\mathrm{t}}\right)$ : $\mathrm{VEF}_{0,5}, \mathrm{VEF}_{0,75} \mathrm{y} \mathrm{VEF}_{1}$, Flujos espiratorios forzados (FEF) al 50\% y entre 25 y $75 \%$ de la CVF $\left(\mathrm{FEF}_{50}\right.$ y $\mathrm{FEF}_{25-75}$ respectivamente), y flujo espiratorio máximo (FEM), relaciones: $\mathrm{VEF}_{1} / \mathrm{CVF}, \mathrm{VEF}_{0,5} / \mathrm{CVF}$, $\mathrm{VEF}_{0,75} / \mathrm{CVF}$. Para los análisis descriptivos, comparativos y elaboración de fórmulas predictivas se utilizaron los mejores valores absolutos obtenidos en volúmenes y flujos espiratorios. Se analizó la calidad de las espirometrías de forma independiente por 2 de los autores (AM y SC), basados en los criterios de Gatto y cols ${ }^{19}$. Las que no cumplían con 3 o más criterios de aceptabilidad se consideraron de mala calidad y debían descartarse del estudio. En caso de discrepancia se llegó a acuerdo.

\section{Comité de Ética}

Este trabajo fue aprobado por el comité de ética de la Pontificia Universidad Católica de Chile ( $\mathrm{N}^{\circ} 15-$ 326). Los padres firmaron consentimiento informado.

\section{Análisis estadístico}

Los datos son presentados como promedio \pm desviación estándar. Se presenta además la diferencia por- centual entre los valores observados y los valores teóricos predeterminados de CVF, $\mathrm{VEF}_{1}, \mathrm{VEF}_{0,5}$ y VEF 0,75. Para la comparación con fórmulas de la GLI (CVF y $\mathrm{VEF}_{1}$ ) además se usó z score, considerando diferencia significativa si este era superior a 0,5 o si la DS era mayor a 1 .

Para evaluar la dispersión de los datos se calculó el coeficiente de variación intraindividual para cada variable ( $\mathrm{CV}=100 \times \mathrm{SD} /$ promedio). Para determinar el grado de asociación lineal entre variables numéricas se usó el coeficiente de correlación de Pearson. Para la comparación de promedios entre grupos se usó test $\mathrm{t}$ de Student para muestras independientes y para la comparación entre valores observados y teóricos se usó test $\mathrm{t}$ de Student para muestras pareadas.

Se elaboraron ecuaciones predictivas para las variables de volúmenes y flujos espiratorios forzados, mediante análisis de regresión múltiple y se determinó el límite inferior de lo normal (LIN), que corresponde al percentil 5 de la recta de regresión ajustada.

Todos los análisis se hicieron usando el programa estadístico SPSS 17, los valores teóricos GLI se obtuvieron mediante macros disponibles para el programa estadístico SAS. Se consideró significativo todo valor $\mathrm{p}$ inferior a 0,05 .

\section{Resultados}

En la Figura 1 se muestra el diagrama de flujo para obtención de espirometrías.

Entre mayo 2016 y agosto 2017 se realizaron 276 espirometrías basales, en niños cuyo rango de edad fue entre 3 años 3 meses y 5 años 11 meses 29 días y talla entre 94 y $129 \mathrm{~cm}$. Las características generales de los sujetos se detallan en la Tabla 1 . No hubo diferencias en peso y talla entre ambos géneros.

En relación a los resultados espirométricos, el promedio de intentos fue de 8 por niño. Se observó mayor éxito de maniobras a mayor edad (Tabla 2), 18 niños (9\%) lograron esfuerzos aceptables en una segunda citación.

Para verificar concordancia entre evaluadores, se analizaron las 276 espirometrías iniciales. Las 74 eliminadas resultaron de mala calidad según ambos evaluadores (100\% concordancia). Hubo discrepancia en los siguientes criterios de aceptabilidad: 1 . Ascenso de la curva F/V 1\%, 2. FEM definido 1\%, 3.descenso irregular 2,5\%, 4. Final de la espiración 0,5\%. Estas espirometrías fueron revisadas luego por ambos evaluadores en conjunto, siendo incluidas para el análisis.

El volumen de extrapolación retrógrada fue menor a $12,5 \%$ (u $80 \mathrm{ml}$ ) en el total de espirometrías seleccionadas. El tiempo espiratorio promedio fue 1,4 segundos $\pm 0,46$ (rango $0,7-2,8$ ) sin diferencia por género. 
Figura 1. Diagrama de flujo de obtención de espirometrías.

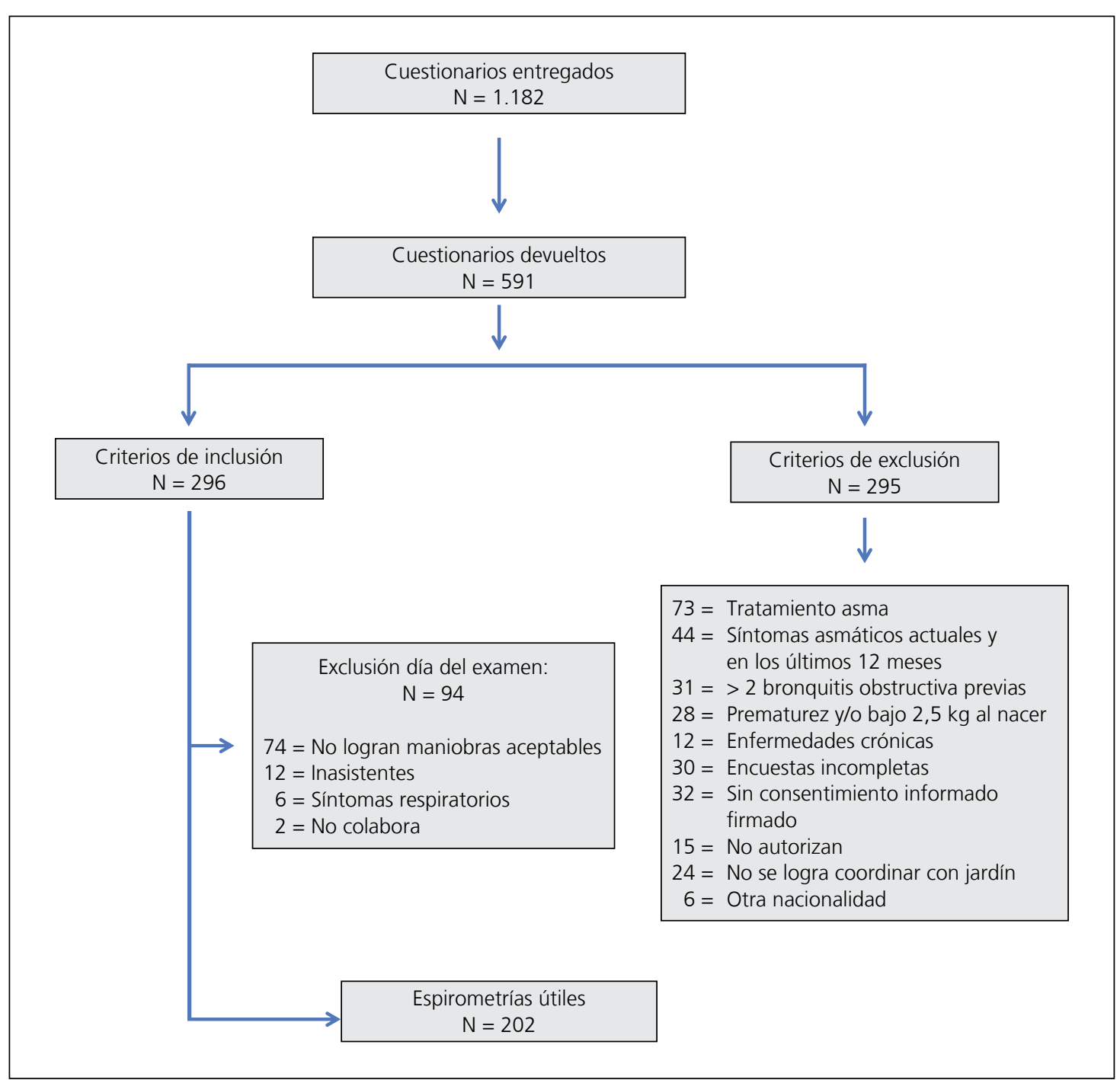

Tabla 1. Descripción general del grupo de estudio $(n=202)$

\begin{tabular}{lc}
\hline Variable & $\mathrm{n}$, promedio \pm DS, porcentaje (\%) \\
\hline Género femenino $\mathrm{n}(\%)$ & $112(55,4)$ \\
Edad promedio (años, DS) & $5,01 \pm 0,57$ \\
3 años a 3 años 11 meses: $\mathrm{n}(\%)$ & $7(3,4)$ \\
4 años a 4 años 11 meses: $\mathrm{n}(\%)$ & $86(42,6)$ \\
5 años a 5 años 11 meses: $\mathrm{n}(\%)$ & $109(54)$ \\
Talla (cm) (promedio \pm DS) & $108 \pm 5,6$ \\
Peso (kg) (promedio \pm DS) & $20 \pm 3,2$ \\
IMC (promedio \pm DS) & $16,8 \pm 1,8$ \\
Estado nutricional & \\
Normopeso $\mathrm{n}(\%)$ & $119(58,9)$ \\
Sobrepeso $\mathrm{n}(\%)$ & $38(18,8)$ \\
Obesidad $\mathrm{n}(\%)$ & $45(22,3)$ \\
Exposición tabaquismo $\mathrm{n}(\%)$ & $54(26,7)$ \\
Historia familiar de asma $\mathrm{n}(\%)$ & $25(12,4)$ \\
\hline
\end{tabular}

DS: desviación estándar. IMC: Indice de masa corporal.
No se observó diferencia significativa en el tiempo espiratorio entre los niños de 4 y 5 años pero si entre los niños de 4 y 5 años con los de 3 años (p 0,04). Los niños (as) que usaron pinza nasal espiraron menos tiempo que quienes no la usaron $(1,37 \pm 0,44$ vs $1,57 \pm 0,50$ segundos, $\mathrm{p}<0,01)$, sin embargo no hubo diferencia en el valor de $\operatorname{CVF}(1,23 \pm 0,21$ vs $1,21 \pm 0,23$ litros, p 0,55$)$. Con el uso de incentivo el tiempo espiratorio fue menor que al no usarlo $(1,37 \pm 0,43$ vs $1,57 \pm 0,52$ segundos, $\mathrm{p}<0,01)$, y la CVF tampoco mostró diferencia $(1,21 \pm 0,21$ vs $1,26 \pm 0,24$ litros, $p$ 0,17).

Veinte niños no alcanzaron una espiración de 1 segundo, por lo tanto no registraron $\mathrm{VEF}_{1}$, se consideraron para cálculos de $\mathrm{VEF}_{0,5}$ y $\mathrm{VEF}_{0,75}$ según el tiempo espiratorio obtenido.

En 56 sujetos se obtuvo solo 1 esfuerzo aceptable, en 1152 , y en 31 3, aceptables y repetibles. En los casos en que se contaba con al menos 2 esfuerzos acep- 
tables se calcularon los coeficientes de variación: CVF: $3,57 \%, \mathrm{VEF}_{1} 3 \%, \mathrm{VEF}_{0,5} 3,26 \%$ y $\mathrm{VEF}_{0,75} 3,1 \%$.

Las variables espirométricas promedio del grupo se describen en la Tabla 3. La CVF fue mayor en los niños de 5 años con respecto a los de 3 y 4 años $(\mathrm{p}<0,001)$. Al comparar por género se observó que solo CVF mostró ser significativamente mayor en los hombres que en mujeres $(1,26 \pm 0,221$ vs $1,20 \pm 0,211$ respectivamente, $\mathrm{p}<0,02)$. En el resto de las variables no hubo diferencia por género (Anexo 1). En la figura 2 se grafica la relación talla vs volúmenes espiratorios forzados.

En la comparación con valores teóricos según GLI, Eigen y França se observó que según porcentaje, los obtenidos en nuestro estudio fueron significativamente mayores, salvo CVF con Eigen (Tabla 3). En la comparación con GLI usando z score la diferencia en CVF fue 0,44 DS y con $\mathrm{VEF}_{1}$ 0,62 DS. Siguiendo
Tabla 2. Porcentaje de éxito en espirometría según edad

\begin{tabular}{lccc}
\hline Edad & $\begin{array}{c}\text { Espirometrías } \\
\text { realizadas (n) }\end{array}$ & $\begin{array}{c}\text { Espirometrías } \\
\text { aceptables (n) }\end{array}$ & $\begin{array}{c}\text { Porcentaje } \\
\text { de éxito * }\end{array}$ \\
\hline 3 a 3 años 11 meses & 24 & 7 & $29 \%$ \\
4 a 4 años 11 meses & 112 & 86 & $76,7 \%$ \\
5 a 5 años 11 meses & 140 & 109 & $77,8 \%$ \\
Total grupo & 276 & 202 & \\
\hline$* 0$ 0,001 comparados entre grupos. &
\end{tabular}

los objetivos de nuestro estudio se elaboraron ecuaciones de referencia para promedio y Percentil 5, las cuales se muestran en la Tabla 4. Para CVF se realizan fórmulas separadas por género por las diferencias encontradas.

Tabla 3. Variables espirométricas promedio grupo estudio, y diferencia porcentual de grupo estudio con respecto a teóricos según GLI, Eigen y França

\begin{tabular}{|c|c|c|c|c|}
\hline Variable (teórico promedio) & Estudio actual & $\begin{array}{c}\text { GLI } \\
\text { \% diferencia }\end{array}$ & $\begin{array}{c}\text { Eigen } \\
\% \text { diferencia }\end{array}$ & $\begin{array}{c}\text { França } \\
\% \text { diferencia }\end{array}$ \\
\hline CVF (I) & $1,22 \pm 0,22$ & $\begin{array}{l}1,15 \pm 0,15 \\
4,6 \pm 10 \%\end{array}$ & $\begin{array}{c}1,23 \pm 0,19 * \\
-1,2 \pm 10,5 \%\end{array}$ & $\begin{array}{c}1,16 \pm 0,15 \\
4,52 \pm 10,4 \%\end{array}$ \\
\hline $\operatorname{VEF}_{1}(\mathrm{I})$ & $1,16 \pm 0,18$ & $\begin{array}{l}1,07 \pm 0,12 \\
6,5 \pm 8,9 \%\end{array}$ & $\begin{array}{c}1,09 \pm 0,14 \\
5,6 \pm 9 \%\end{array}$ & $\begin{array}{c}1,07 \pm 0,16 \\
6,08 \pm 9,47 \%\end{array}$ \\
\hline $\operatorname{VEF}_{0,5}(\mathrm{I})$ & $0,91 \pm 0,15$ & - & - & $\begin{array}{c}0,80 \pm 0,09 \\
10,55 \pm 10,34 \%\end{array}$ \\
\hline $\operatorname{VEF}_{0,75}(\mathrm{I})$ & $1,07 \pm 0,17$ & $\begin{array}{l}0,99 \pm 0,11 \\
5,8 \pm 9,6 \%\end{array}$ & - & $\begin{array}{l}- \\
-\end{array}$ \\
\hline
\end{tabular}

*p 0,8. Resto de comparaciones $p<0,01$. CVF: Capacidad vital forzada, VEF: Volúmen espirado forzado.

Tabla 4. Ecuaciones para cálculo de valor promedio y valor límite inferior de variables espirométricas para niños en edad pre-escolar

\begin{tabular}{|c|c|c|c|c|c|}
\hline \multirow[b]{2}{*}{ Variable } & \multicolumn{2}{|c|}{ Ecuación para Valor Medio } & \multirow[b]{2}{*}{$R^{2}$} & \multicolumn{2}{|c|}{ Ecuación para LIN } \\
\hline & Constante & Talla & & Constante & Talla \\
\hline VEF1 & $-1,602$ & 0,025 & 0,60 & $-1,794$ & 0,025 \\
\hline CVF Hombres & $-2,058$ & 0,031 & 0,61 & $-2,304$ & 0,031 \\
\hline CVF Mujeres & $-2,125$ & 0,031 & 0,65 & $-2,320$ & 0,031 \\
\hline $\mathrm{VEF}_{1} / \mathrm{CVF}$ & 115,643 & $-0,204$ & 0,05 & 107,4 & $-0,204$ \\
\hline $\mathrm{VEF}_{0,5}$ & $-1,151$ & 0,019 & 0,51 & $-1,323$ & 0,019 \\
\hline $\mathrm{VEF}_{0,5} / \mathrm{CVF}$ & 110,003 & $-0,323$ & 0,06 & 98,262 & $-0,323$ \\
\hline $\mathrm{VEF}_{0,75}$ & $-1,446$ & 0,023 & 0,57 & $-1,631$ & 0,023 \\
\hline $\mathrm{VEF}_{0,75} / \mathrm{CVF}$ & 116,1 & $-0,264$ & 0,05 & 105,728 & $-0,264$ \\
\hline $\mathrm{FEF}_{25-75}$ & $-1,100$ & 0,025 & 0,15 & $-1,652$ & 0,025 \\
\hline PEF & $-2,943$ & 0,051 & 0,36 & $-3,584$ & 0,051 \\
\hline
\end{tabular}

R2: coeficiente de determinación. LIN: límite inferior de normalidad, corresponde a Percentil 5. Para calcular el valor promedio se usa la siguiente fórmula: Constante + (coeficiente talla $\mathrm{x}$ talla [en cm]). Para calcular LIN: Constante + (coeficiente talla x talla [en cm]). CVF: Capacidad vital forzado; VEF: Volúmen espirado forzado; FEF: Flujo espiratorio forzado. 


\section{Discusión}

En este estudio obtuvimos una muestra de 202 espirometrías realizadas en niños chilenos sanos en edad pre-escolar. Al comparar sus resultados con teóricos extranjeros se encontró diferencia significativa en la gran mayoría de las variables espirométricas. Se construyeron y se proponen ecuaciones predictivas para evaluar volúmenes y flujos espiratorios forzados en este grupo etario.

Los niños evaluados pertenecían a jardines infantiles y colegios de diversas comunas de Santiago, de diferente nivel socioeconómico. Se obtuvo un alto porcentaje de respuesta a las encuestas. El peso y talla no variaron entre hombres y mujeres lo que es habitual en edad preescolar. En relación al estado nutricional, el grupo presenta un porcentaje de obesidad similar, y para sobrepeso algo menor que lo descrito en cifras nacionales del año $2016^{20}$. A pesar de esto encontramos que la CVF fue mayor que la predicha por las fórmulas extranjeras. En general al medir función pulmonar en niños sanos se exige estén asintomáticos por al menos 3 semanas $^{1}$. En nuestro estudio se incluyeron niños que presentaban síntomas respiratorios altos leves, basado en el estudio de Lum et al ${ }^{18}$ el cual demostró que su presencia no influía en las mediciones.

En relación a nuestro primer objetivo, se logró un porcentaje de éxito de 73,2\% (202 de 276, Figura 1), semejante a varias casuísticas descritas previamente ${ }^{3,5,7,8}$, sin embargo hay publicaciones que muestran valores tan altos como $82-88 \%^{9,21}$. Como se esperaba, a mayor edad mayor éxito de la maniobra, igual que otras experiencias ${ }^{10,23,24}$. Destaca el 91\% de los niños (as) lograron curvas aceptables en la primera visita, superior a lo descrito en otras series 5 , una explicación de esto podria ser la realización del examen en grupos de máximo 3 niños, lo que permitía una explicación de la maniobra personalizada y adecuada a la edad, en un ambiente grato $^{24}$. Por otro lado también podría explicarse por el uso de incentivo, en el 75,7\% de los niños, cuando

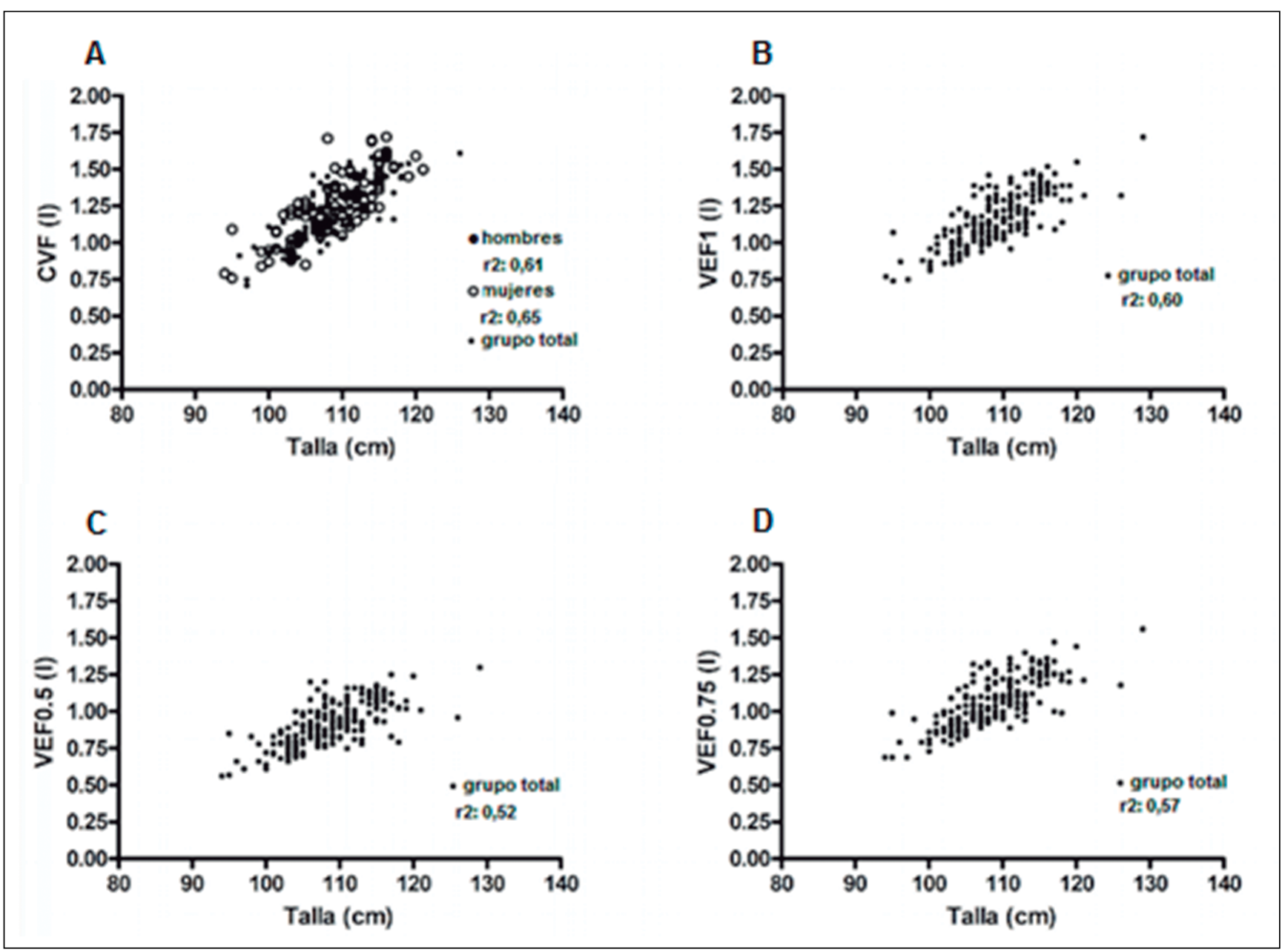

Figura 2. Relación talla vs volúmenes espiratorios forzados. A: relación talla/CVF en hombres y mujeres separado. B, C y D: Relación talla/NEF 1 , talla/ $V_{E F} F_{0,5}$ y talla $V E F_{0,75}$ respectivamente, en grupo total. CVF: Capacidad Vital Forzada; $V E F_{1}$ : Volumen Espirado Forzado al primer segundo; VEF 0,5 : volumen espirado forzado al 0,5 segundo; $\mathrm{VEF}_{0,75}$ : Volumen Espirado forzado al 0,75 segundo. 
se notaba que el niño requería mayor estímulo o no entendía las instrucciones. El tipo de animación más utilizado incentivaba a alcanzar un Flujo Espiratorio Máximo (FEM) adecuado y luego prolongar la espiración hasta alcanzar el volumen residual. Sin embargo, su uso acortó el tiempo espiratorio, pero sin afectar la medición de CVF. Aún es controvertida esta herramienta, probablemente el niño se distrae y se afecta el esfuerzo espiratorio. Gracchi et al compararon el uso y no uso de incentivo en niños de 4-5 años, encontrando que se obtenía una menor repetibilidad en CVF y VEF1 al usarlo ${ }^{25}$. Chavasse et al vieron que no usar pinza nasal no afecta las mediciones de CVF ni VEF1 en niños en edad escolar ${ }^{26}$. En nuestra serie su uso también acortó el tiempo espiratorio, pero tampoco hubo diferencia en la medición de CVF. En un estudio español el 69,2\% usó pinza nasal, sin observarse disminución del tiempo espiratorio ${ }^{4}$.

Las espirometrías utilizadas para los análisis cumplían con los criterios de aceptabilidad sugeridos por la ATS/ERS $2007^{1}$, y mostraban muy bajos coeficientes de variación entre las mediciones. Se seleccionaron para los análisis solo los exámenes valorados como de "muy buena calidad"19. También se consideraron 20 espirometrías que tenían tiempo espiratorio menor a 1 segundo, utilizando los valores de $\mathrm{VEF}_{0,5}$ y $\mathrm{VEF}_{0,75}$ para sus fórmulas predictivas correspondientes. Los niños menores de 6 años aún tienen su pulmón en etapa de crecimiento, las vías aéreas son relativamente más grandes que el parénquima pulmonar, lo que explicaría un vaciamiento rápido con la espiración forzada, con un final brusco y precoz $z^{8,11,27}$. Los niños a esta edad pueden expresar su CVF con espiraciones menores a 1 segundo, obteniendo valores de $\mathrm{VEF}_{0,5}$ y $\mathrm{VEF}_{0,75}$, los cuales tienen buena reproducibilidad y son útiles para evaluar respuesta a broncodilatador ${ }^{28-30,37}$.

Actualmente contamos con numerosas publicaciones de patrones de referencia para niños en edad pre-escolar ${ }^{1,2,10,14-17}$, sin embargo, para poder aplicarlas en nuestra población debiéramos encontrar similitud con alguno de ellos en las mediciones de al menos 300 niños de la misma edad ${ }^{1}$. Decidimos realizar comparaciones con GLI por tratarse de la recomendación actual ${ }^{31}$ y porque permite continuidad para interpretar las espirometrías de una persona ya que abarca desde los 3 hasta los 95 años. Quisimos comparar también con Eigen ${ }^{2}$ por tratarse de los valores de referencia utilizados en nuestro laboratorio. Esto debido a haber realizado un ejercicio comparativo el año 2011, y encontrar que estos valores teóricos eran los que mayormente semejaban las mediciones realizadas en niños chilenos (32, no publicado). Comparamos con la publicación de Brasil ${ }^{10}$ por ser la serie de mayor n para elaborar ecuaciones en América del Sur. Encontramos diferencias significativas en todas las variables salvo CVF con Eigen. Estas diferencias podrían explicarse: 1) por el tamaño muestral ${ }^{33}$, siendo el nuestro semejante al de Eigen y França; 2) Por el neumotacógrafo utilizado, y la posición al realizar la espirometría, de pie o sentado ${ }^{34,35}$; 3) Aunque en niños de esta edad no hay estudios, podría explicarse por diferencias étnicas ${ }^{35}$, ya que los teóricos de GLI se basan en mediciones en niños caucásicos, los de Eigen en niños norteamericanos, y los de França en niños de Sete Lagoas, Brasil. En el estudio español de Martin y cols también encuentran diferencias porcentuales importantes entre promedios teóricos GLI y Cande$\mathrm{la}^{4}$, en nuestro estudio llama la atención que nuestros valores sean superiores en la mayoría de las variables a los medidos por França y Eigen, siendo que ellos incluyen niños de 6 años. Debe notarse sí que las diferencias en valores absolutos es muy pequeña por tratarse de volúmenes pulmonares que aproximan 1 litro en CVF. En este sentido pudimos realizar comparación con las fórmulas GLI mediante z score, mostrando que no había diferencia significativa. Al realizar regresiones múltiples, se encontró que la talla fue el factor más determinante de cada variable de función pulmonar, al igual que se ha encontrado en estudios previos ${ }^{4,10,13,16,27}$. También, en forma semejante a estos estudios, los coeficientes de correlación $\left(\mathrm{r}^{2}\right)$ resultaron moderados entre talla y volúmenes y bajos para relaciones $\mathrm{VEF}_{\mathrm{t}} / \mathrm{CVF}$ y flujos espiratorios forzados (Tabla 4). Se proponen así fórmulas predictivas para niños en edad pre-escolar, para valor promedio y valor límite inferior (Percentil 5), las cuales se muestran en la Tabla 4.

Consideramos debilidades del estudio el no haber logrado un mayor número de espirometrías en niños de 3 años. A esta edad es mayor la dificultad para obtener esfuerzos aceptables, y por otro lado es más difícil conseguir voluntarios ya que en general a esta edad no están escolarizados. Otra debilidad podría ser que el n de 202 sería insuficiente ${ }^{33}$, explicando los coeficientes de correlación moderados y parte de las diferencias con otros valores teóricos, aunque un mayor tamaño muestral no asegura encontrar mejores resultados.

\section{Conclusiones}

La espirometría forzada basal en niños pre-escolares residentes en Santiago puede realizarse adecuadamente y con éxito en un gran porcentaje de los casos. Hemos obtenido valores de normalidad en una población sana siguiendo estrictamente la normativa actual publicada por la ATS/ERS 2007. Proponemos el uso de estas fórmulas para la interpretación de espirometrías de pacientes entre 3 y 5 años 11 meses con enfermedades respiratorias residentes en Chile. En su defecto po- 
drían también utilizarse las de GLI, ya que sus diferencias no son significativas. Estas últimas fórmulas tienen la ventaja de abarcar en forma continua el cálculo de valores teóricos desde los 3 hasta los 95 años.

\section{Responsabilidades Éticas}

Protección de personas y animales: Los autores declaran que los procedimientos seguidos se conformaron a las normas éticas del comité de experimentación humana responsable y de acuerdo con la Asociación Médica Mundial y la Declaración de Helsinki.

Confidencialidad de los datos: Los autores declaran que han seguido los protocolos de su centro de trabajo sobre la publicación de datos de pacientes.

Derecho a la privacidad y consentimiento informado: Los autores han obtenido el consentimiento informado de los pacientes y/o sujetos referidos en el artículo. Este documento obra en poder del autor de correspondencia.

\section{Conflicto de intereses}

Los autores declaran no tener conflicto de intereses.

\section{Agradecimientos}

Se agradece la valiosa colaboración de directores, apoderados, parvularias, auxiliares de párvulo, y especialmente de los niños de los siguientes establecimientos educacionales: Colegio Blas Cañas, Colegio Huelén, Colegio Kent, Colegio Villa María, Jardín Infantil Barco de Papel, Jardin Greenfield, Colegio San Marcos, Colegio El Bosque, Instituto O’Higgins, Jardín Pintamigos, Jardines Infantiles San Joaquin y Campus Oriente de la P. Universidad Católica de Chile

\section{Financiamiento}

Fondos concursables Sociedad Chilena de Neumología Pediátrica.

Anexo 1. Valores promedio de variables espirométricas en mujeres y hombres

\begin{tabular}{|c|c|c|c|c|c|c|c|c|}
\hline Género (n) & $\begin{array}{l}\text { CVF } \\
(\mathrm{L})\end{array}$ & $\begin{array}{c}\mathrm{VEF}_{1} \\
(\mathrm{~L})\end{array}$ & $\begin{array}{c}\text { CVFNEF }{ }_{1} \\
(\%)\end{array}$ & $\begin{array}{l}\mathrm{VEF}_{0,5} \\
(\mathrm{~L})\end{array}$ & $\begin{array}{c}\operatorname{VEF}_{0,5} / \mathrm{CVF} \\
(\%)\end{array}$ & $\begin{array}{c}\operatorname{VEF}_{0,75} \\
(\mathrm{~L})\end{array}$ & $\begin{array}{c}\mathrm{FEF}_{25-75} \\
(\mathrm{~L} / \mathrm{s})\end{array}$ & $\begin{array}{l}\text { FEM } \\
(\mathrm{L} / \mathrm{s})\end{array}$ \\
\hline Masculino (90) & $1,26 \pm 0,22^{*}$ & $1,18 \pm 0,18$ & $93 \pm 5,3$ & $0,92 \pm 0,15$ & $73,5 \pm 7,46$ & $1,09 \pm 0,17$ & $1,56 \pm 0,35$ & $2,67 \pm 0,50$ \\
\hline Femenino (112) & $1,20 \pm 0,21 *$ & $1,14 \pm 0,17$ & $93,6 \pm 4,8$ & $0,90 \pm 0,14$ & $75,9 \pm 7,07$ & $1,05 \pm 0,16$ & $1,59 \pm 0,36$ & $2,62 \pm 0,47$ \\
\hline Promedio grupo & $1,22 \pm 0,22$ & $1,16 \pm 0,18$ & $93,3 \pm 5,07$ & $0,91 \pm 0,15$ & $74,9 \pm 7,32$ & $1,07 \pm 0,17$ & $1,58 \pm 0,36$ & $2,64 \pm 0,48$ \\
\hline
\end{tabular}

${ }^{*} p<0,01$. CVF: capacidad vital forzada; VEF: volumen espiratorio forzado; FEF: Flujo espiratorio forzado; FEM: flujo espiratorio máximo.

\section{Referencias}

1. Beydon N, Davis SD, Lombardi E, et al. An Official AmericanThoracic Society/ European Respiratory Society Statement: Pulmonary Function Testing in Preschool Children. Am J Respir Crit Care Med. 2007; 175:1304-13452.

2. Eigen H, Bieler H, Grant D, et al. Spirometric pulmonary function in healthy preschool children. Am J Respir Crit Care Med. 2001; 163:619-23.

3. Neves T, Araujo L. Feasibility of spirometry in preschool children. J Bras Pneumol. 2011;37(1):69-74.

4. Pérez-Yarza G, Villa JR, Cobos N, et al. Espirometría forzada en preescolares sanos bajo las recomendaciones de la ATS/ERS: Estudio CANDELA. An Pediatr (Barc). 2009; 70:3-11.

5. Kampschmidt J, Brooks E, Cherry D, Guajardo J, Wood P. Feasibility of Spirometry Testing in Preschool Children. Pediatr Pulmonol. 2016; 51:258-66.
6. Kozlowska W, Aurora P. Spirometry in the pre-school age group. Paediatr Respir Rev. 2005; 6:267-72.

7. Gaffin JM, Lichtenberg N, Martin TR, Phipatanakul W. Clinically useful spirometry in preschool-aged children: evaluation of the 2007 American Thoracic Society guidelines. J Asthma. 2010;47:7627.

8. Burity EF, De Castro Pereira CA, Rizo JA, Cavalcanti Sarinho ES, Jones MH. Early termination of exhalation: Effect on spirometric parameters in healthy preschol children. J Bras Pneumol. 2001;37:464-470.

9. Veras TN, Pinto LA. Feasibility of spirometry in preschool children. J Braz Pneumol. 2011;37:69-74.

10. Franca DC, Camargos PA, Jones MH, et al. Prediction equations for spirometry in four- to six-year-old children. J Pediatr (Rio J). 2016;92:400-11.

11. Donaire R, González S, Moya A, Fierro L, Brockmann P, Caussade S.
Spirometry interpretation feasibility among pre-school children according to the European Respiratory Society and American Thoracic Society Guidelines. Rev Chil Pediatr. 2015;86(2):86-91.

12. Stocks J. Clinical implications of pulmonary function testing in preschool children. Paediatr Respir Rev. 2006;7S:S26-9.

13. Coates AL. Using reference values to interpret pulmonary function tests. Paediatr Respir Rev. 2011;12:206-7.

14. Quanjer PH, Stanojevic S, Cole TJ, Baur X, Hall GL, Culver BH, Enright PL,Hankinson JL, Ip MSM, Zheng J, Stocks J and the ERS Global Lung Function Initiative. Multi-ethnic reference values for spirometry for the 3-95-yr age range: the global lung function 2012 equations. Eur Respir J. 2012; 40: 1324-43.

15. Zapletal A, Chapulova J. Forced expiratory parameters in healthy preschool children (3-6 years of age). Pediatr Pulmonol. 2003;35:200-7. 
16. Nystad W, Samuelsen SO, Nafstad P, Edvardsen E, Stensrud T, Jaakkola JJK. Feasibility of measuring lung function in preschool children. Thorax. 2002;57:10217.

17. Stanojevic S, Wade A, Cole TJ, et al. Spirometry Centile Charts for Young Caucasian Children. The Asthma UK Collaborative Initiative. Am J Respir Crit Care Med. 2009;180:547-52

18. Lum S, Bountziouka V, Sonnappa S, Cole T, Bonner R and Stocks J. How "healthy" should children be when selecting reference samples for spirometry? Eur Respir J. 2015;45:1576-81.

19. Gatto F, Bedregal P, Ubilla C, Barrientos $\mathrm{H}$, Caussade S. Elaboration of a quality scale for the interpretation of spirometry in preschool children. Rev Chil Pediatr. 2017;88(1):58-65.

20. Mapa nutricional 2016. JUNAEB marzo 2017. www.junaeb.cl.

21. Santos N, Almeida I, Couto M, MoraisAlmeida M, Borrego LM. Feasibility of routine respiratory function testing in preschool children. Rev Port Pneumol. 2013;19(1):38-41.

22. Martín de Vicente C, de Mir Messa I, Rovira Amigo S, et al. Validación de las ecuaciones propuestas por la Iniciativa Global de Función Pulmonar (GLI) y las de Todas las Edades para espirometría forzada en preescolares sanos españoles. Arch Bronconeumol. 2018; 54(1):24-30.

23. Vilozni D, Barak A, Efrati O, et al. The Role of Computer Games in Measuring
Spirometry in Healthy and "Asthmatic" Preschool Children. Chest 2005; 128:1146-55.

24. Aurora P, Stocks J, Oliver C, et al, on behalf of the London Cystic Fibrosis Collaboration. Quality Control for Spirometry in Preschool Children with and without Lung Disease. Am J Respir Crit Care Med. 2004;169:1152-9.

25. Gracchi V, Boel M, van der Laag J, van der Ent CK. Spirometry in young children: should computer-animation program be used during testing? Eur Respir J. 2003;21:872-5.

26. Chavasse R, Johnson P, Francis J, BalfourLynn I, Rosenthal M, Bush A. To clip or not to clip. Noseclips for spirometry. Eur Respir. J 2003; 21: 876-8

27. Piccioni1 $\mathrm{P}$, Tassinari $\mathrm{R}$, Carosso A, Carena C, Bugianil M, Bono R. Lung function changes from childhood to adolescence: a seven-year follow-up study. BMC Pulm Med. 2015;15:31-8.

28. Burity EF, Pereira CA, Jones MH, Sayão LB, Andrade AD, Britto MC. Bronchodilator response cut-off points and FEV0.75 reference values for spirometry in preschoolers. J Bras Pneumol. 2016 ;42(5):326-32.

29. Borrego LM, Stocks J, Almeida I, et al. Bronchodilator responsiveness using spirometry in healthy and asthmatic preschool children. Arch Dis Child. 2013;98(2):112-7.

30. Busi LE, Restuccia S, Tourres R, Sly PD. Assessing bronchodilator response in preschool children using spirometry. Thorax. 2017;72:367-72.

31. Culver BH, Graham BL, Coates AL, et al. Recommendations for a Standardized Pulmonary Function Report. An Officia American Thoracic Society Technical Statement Am J Respir Crit Care Med. 2017;196(11):1463-72.

32. Pizarro ME, Navarrete P, Cerda J, Caussade S. Espirometría en pre-escolares chilenos: ¿qué valores de referencia debemos usar? Resúmenes V Congreso Sociedad Chilena de Neumología Pediátrica. Neumol Pediatr. 2009;4(2):95.

33. Quanjer PH, Stocks J, Cole TJ, Hall GL, Stanojevic S. Influence of secular trends and sample size on reference equations for lung function tests. Eur Respir. J 2011; 37: 658-64.

34. Townsend MC. Spirometric forced expiratory volumes measured in the standing versus the sitting posture. Am Rev Respir Dis. 1984;130(1):123-4.

35. Swamy K, Isroff C, Mhanna MJ, Chouksey AK. Effect of sitting vs standing posture on spirometry in children. Ann Allergy Asthma Immunol. 2016;117(1):94-6.

36. Strippoli MPF, Kuehni CE, Dogaru CM, et al. Etiology of Ethnic Differences in Childhood Spirometry. Pediatr. 2013;131:e1842-9.

37. Linares M, Meyer R, Contreras I, Delgado I, Castro-Rodríguez JA. Utility of bronchodilator response for asthma diagnosis in Latino preschoolers Allergol Immunopathol (Madr). 2014;42(6):553-9. 